\title{
The Effect of Noise on Transverse Emittance Growth in the Tevatron
}

\author{
C.Y. Tan*, FNAL, Batavia, IL 60510, USA \\ J.M. Steimel ${ }^{\dagger}$, FNAL, Batavia, IL 60510, USA
}

\section{Abstract}

Emittance growth due to noise from a transverse beam feedback system are discussed. A theory for calculating emittance growth rate as a function of the feedback system's measured open loop transfer function is derived. A simple feedback system was installed, measured, and tested in the Fermilab Tevatron, and the emittance growth rate results agree very closely with the theory.

\section{INTRODUCTION}

As particle densities in accelerators and storage rings increase, instabilities arise that dilute emittance or, at worst, actually drive the beam out of the machine. Beam feedback systems can extend the intensity threshold of these instabilities. These systems have pushed the intensity limits of many accelerators beyond their design intensity and have become a necessity for present day operation.

For most beam feedback applications, the dominant design specification is the system gain required to counteract the instability growth rate. This gain is predominantly limited by the delay and dynamic range of the system. A secondary but significant design specification is the amount of noise power that is deposited in the beam. This noise will cause emittance growth over time periods on the order of minutes unless there is some other damping mechanism such as synchrotron radiation. Noise will have a minimal effect on fast cycling machines, but it could have a devastating effect on hadron storage rings that must maintain high luminosity.

Instrumentation for measuring feedback system response functions has become increasingly powerful in the last four years, and feedback designers have taken advantage of this when designing new feedback systems for existing machines. By measuring the system response at low beam intensity, the stability of the system at higher intensities can be extrapolated. The theory and application of this technique are well documented.

This paper documents the extrapolation of noise properties of the feedback system from the system response measurements. First, a theory for determining relative emittance growth rate as a function of the system response is derived. Second, the theory is tested in the Fermilab Tevatron storage ring. The paper will show how the measured relative growth rates compare between feedback loops open and closed, and it will show how closely these rates compare with theory.

\footnotetext{
*cytan@fnal.gov

† steimel@fnal.gov
}

\section{THEORY}

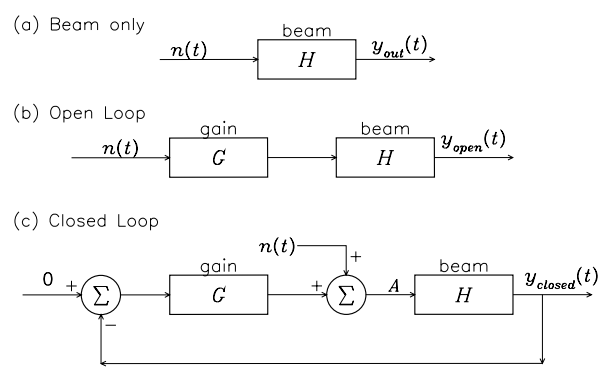

Figure 1: Here are the three systems that will be considered in this section.

In this section, we will derive the relative growth rates of the emittance of the beam when the damper loop is open and when it is closed given its open loop response function $G(\omega) H(\omega)$. Referring to Figure 1(a), if the response function in the time domain is $h(t)=u(t) \bar{h}(t)$ where $u(t)$ is the unit step function, then the output $y_{\text {out }}$ from noise $n(t)$ is simply the convolution

$$
y_{\text {out }}(t)=\int_{0}^{t} d \tau \bar{h}(\tau) n(t-\tau)
$$

where we have used $n(t)=0$ if $t<0$. Then the mean squared growth $\left\langle y_{\text {out }}^{2}\right\rangle$ is simply

$$
\left\langle y_{\text {out }}^{2}\right\rangle=\int_{-\infty}^{\infty} d \omega\left|\int_{0}^{t} d \tau \bar{h}(\tau) e^{i \omega \tau}\right|^{2} S(\omega)
$$

where $S(\omega)$ is the spectral power density function. $S(\omega)$ is defined to be

$$
P_{\mathrm{av}}=\lim _{T \rightarrow \infty} \frac{1}{2 T} \int_{-T}^{T} d t x^{2}(t)=\int_{-\infty}^{\infty} d \omega S(\omega)
$$

and it can be shown [1] by using the stationary property that

$$
\left\langle n\left(t-t^{\prime}\right) n\left(t-t^{\prime \prime}\right)\right\rangle=\int_{-\infty}^{\infty} d \omega S(\omega) e^{-i \omega\left(t^{\prime \prime}-t^{\prime}\right)}
$$

For Figure 1(b), when there is a gain element $G(\omega)$ in the system, we can use (3) and Parseval's theorem to show that

$$
\left\langle y_{\mathrm{open}}\right\rangle^{2}=\int_{-\infty}^{\infty} d \omega\left|\int_{0}^{t} d \tau \bar{h}(\tau) e^{i \omega \tau}\right|^{2}|G(\omega)|^{2} S(\omega)
$$


Finally for Figure 1(c), when we close the loop,

$$
G(\omega) \rightarrow \frac{1}{1+G(\omega) H(\omega)}
$$

and thus

$$
\begin{gathered}
\left\langle y_{\text {closed }}\right\rangle^{2}=\int_{-\infty}^{\infty} d \omega\left|\int_{0}^{t} d \tau \bar{h}(\tau) e^{i \omega \tau}\right|^{2} \times \\
\left|\frac{1}{1+G(\omega) H(\omega)}\right|^{2} S(\omega)
\end{gathered}
$$

If the observed growth rate when $t$ is large is dominated by the linear part of $\left\langle y_{\text {open }}\right\rangle^{2}$ then

$$
\begin{gathered}
d\left\langle y_{\mathrm{open}}\right\rangle^{2} / d t=\text { slope }\left[\int_{-\infty}^{\infty} d \omega\left|\int_{0}^{t} d \tau \bar{h}(\tau) e^{i \omega \tau}\right|^{2} \times\right. \\
\left.|G(\omega)|^{2} S(\omega)\right]
\end{gathered}
$$

where slope[.] is defined to be the slope of the function for large $t$.

If we assume $S(\omega)=$ constant and $G(\omega)=g$, where $g$ is independent of $\omega$, then the relative growth is

$$
\frac{d\left\langle y_{\text {closed }}^{2}\right\rangle / d t}{d\left\langle y_{\text {closed }}^{2}\right\rangle /\left.d t\right|_{g=0}}=\frac{\text { slope }\left[\int_{-\infty}^{\infty} d \omega\left|\frac{\int_{0}^{t} d \tau \bar{h}(\tau) e^{i \omega \tau}}{1+g H(\omega)}\right|^{2}\right]}{\text { slope }\left[\int_{-\infty}^{\infty} d \omega\left|\int_{0}^{t} d \tau \bar{h}(\tau) e^{i \omega \tau}\right|^{2}\right]}
$$

Equation (9) is the equation which we will use to fit the measured data shown in the next section.

\section{EXPERIMENT}

The simplified block diagram of our narrow band damper system is shown in Figure 2. This block diagram is an example of the system shown in Figure 1(c), Thus, we can use the previously discussed theoretical results to analyze the experimental data. When $G$ is set to zero and only noise is

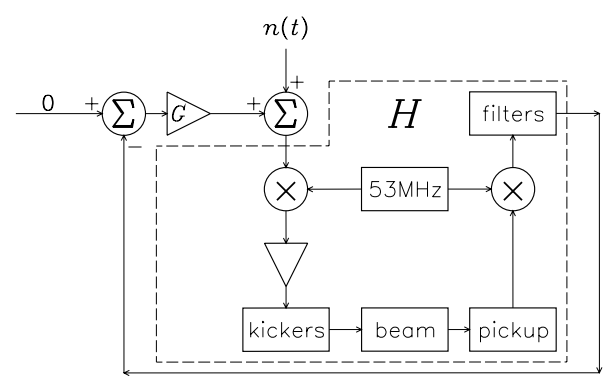

Figure 2: This figure shows a simplified block diagram of our narrow band damper.

fed into the beam at $150 \mathrm{GeV}$, we see that the vertical beam size grows as expected. This is shown in Figure 3. In fact the growth of $\left.\left\langle y_{\text {closed }}^{2}\right\rangle\right|_{G=0}$ is linear as shown in Figure 4. Now, when the loop is closed and $G \approx 5.5$ at $18.327 \mathrm{kHz}$, the growth rate is reduced by a factor of 3 from $(0.71 \pm$ $0.05) \times 10^{-3} \mathrm{~mm}^{2} \mathrm{~s}^{-1}$ to $(0.24 \pm 0.05) \times 10^{-3} \mathrm{~mm}^{2} \mathrm{~s}^{-1}$.

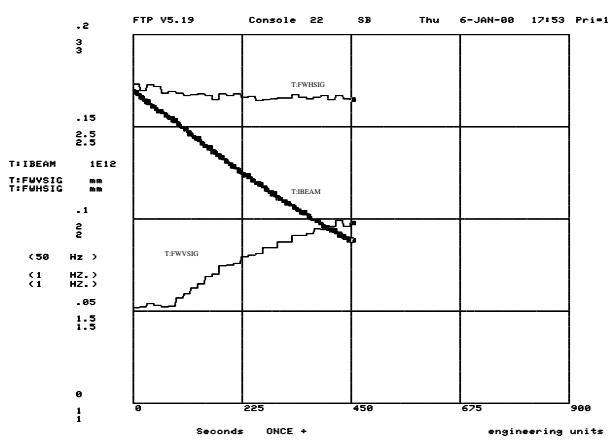

Figure 3: This graph shows how the vertical beam size typically grows when a noisy vertical kicker is used to excite the beam. In this case, the noise density $S_{0}=1.25 \mathrm{~W} / \mathrm{kHz}$ between $17.417 \mathrm{kHz}$ and $19.417 \mathrm{kHz}$.

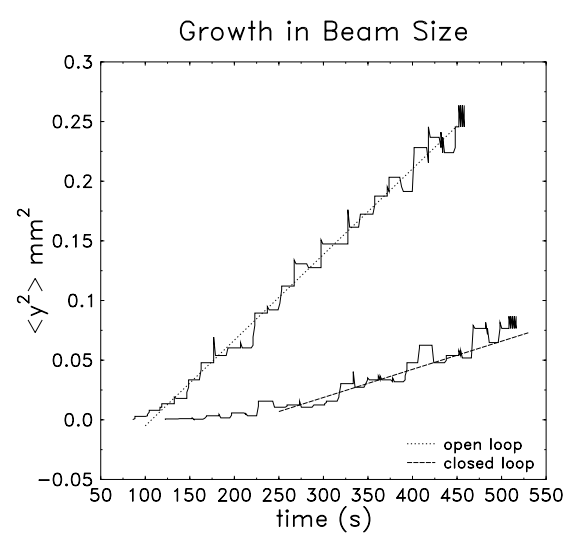

Figure 4: It is clear from here that the growth rate is linear which satisfies the condition required in the theory. When the loop is closed, the growth rate is reduced by a factor of 3.

We plot the measured relative growth rates and the theoretical relative growth rates in Figure 5. The theoretical growth rate was calculated using (9) with the measured $G(\omega) H(\omega)$ shown in Figure 6. It is important to notice that there are no free parameters in the theory, i.e. the theory is not fitted to the data. The goodness of fit criteria is given by

$$
\chi_{\nu}^{2}=\frac{1}{\nu} \sum_{i=1}^{N} \frac{1}{\sigma_{i}^{2}}\left(\frac{d\left\langle y_{\text {expt }}^{2}\right\rangle_{i}}{d t}-\frac{d\left\langle y_{\text {theory }}^{2}\right\rangle_{i}}{d t}\right)^{2}
$$

where $\nu=N-1$ and $N$ is the number of data points. Using (10), the goodness of fit our theory to the measured data is 1.3. According to Bevington [2], $\chi_{\nu}^{2}$ should be about 1 and less than 1.5 for a good fit. As a comparison, we also 
show in this plot a linear least squares fit constrained to pass through $(0,1)$ of the data. The linear least squares plot has a $\chi_{\nu}^{2} \approx 3$.

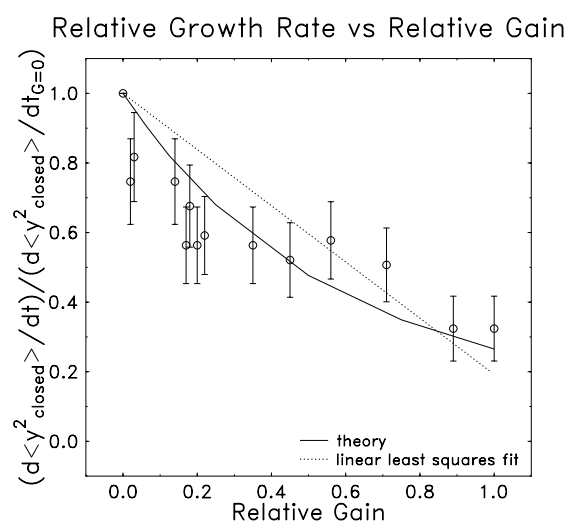

Figure 5: The theoretical curve is shown here with the data. A linear least squares fit of the data which must pass through the point $(0,1)$ is superimposed for comparison.

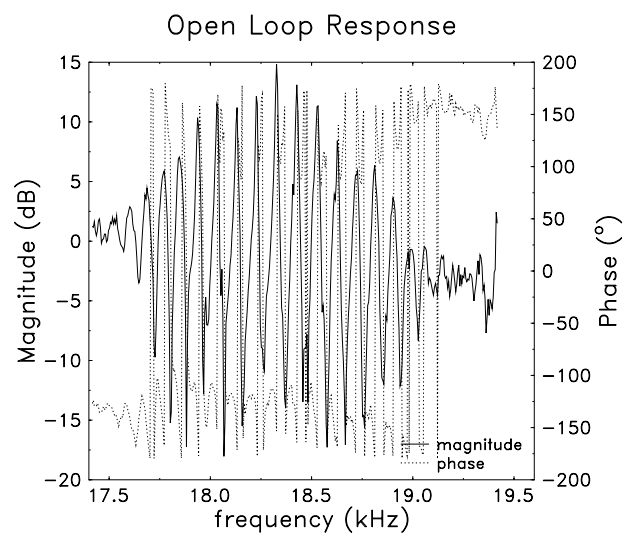

Figure 6: This is the measured open loop response of the system i.e. $G(\omega) H(\omega)$.

Finally the measured absolute growth rate when the loop is open versus the spectral power density is shown in Figure 7. We see that the the growth rate is linear w.r.t. spectral power density. The least squares fit gives the emittance growth rate per spectral power density to be $\left(0.55 \times 10^{-2}\right) \pi\left(\mathrm{mm} \cdot \mathrm{mrad} \cdot \mathrm{s}^{-1}\right) /(\mathrm{W} / \mathrm{kHz})$ or $19.8 \pi\left(\mathrm{mm} \cdot \mathrm{mrad} \cdot \mathrm{hr}^{-1}\right) /(\mathrm{W} / \mathrm{kHz})$ over the narrow band of band of $2 \mathrm{kHz}$ which encloses all the tune lines centred about $18.847 \mathrm{kHz}$. Using this number and assuming that the maximum emittance growth rate is $0.2 \pi \mathrm{mm} \cdot \mathrm{mrad} \cdot \mathrm{mrad}$ $\cdot \mathrm{hr}^{-1}$ allowed in the Tevatron, then the maximum noise density that we can have in the narrow band damper system is $10 \mathrm{~mW} / \mathrm{kHz}$. For a broadband system of $10 \mathrm{MHz}$, we have approximately 425 tune bands in this bandwidth, then the maximum noise density that we can allow in the broadband system is $24 \mu \mathrm{W} / \mathrm{kHz}$ if we assume that each band contributes linearly to the growth rate.

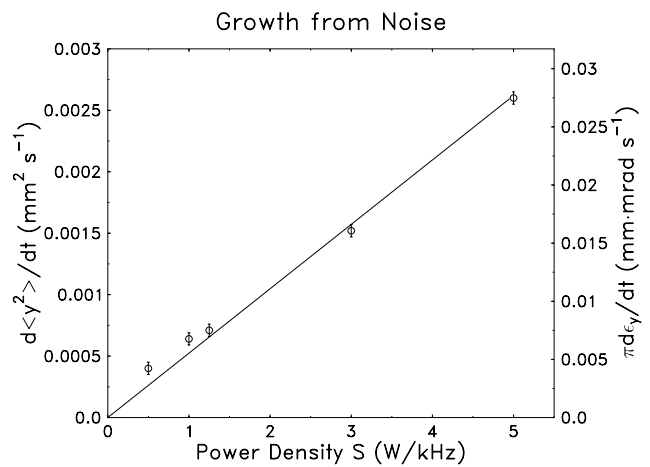

Figure 7: The absolute growth rate at $150 \mathrm{GeV}$ when the loop is open versus the spectral power density is linear. The noise bandwidth is fixed at $2 \mathrm{kHz}$ centred about $18.847 \mathrm{kHz}$.

\section{CONCLUSION}

We have demonstrated how we can calculate the growth rate when we are given the open loop response. This observed relative growth rate agrees with the theoretical relative growth rate. Therefore, if we are given the open loop response of the damper system, we can calculate what the expected growth rate of the beam. Finally, from our measurements of the absolute growth rate we can put an upper limit on the noise density that we are allowed for the damper design.

\section{REFERENCES}

[1] J.S. Bendat, "Principles and Applications of Random Noise Theory”, John Wiley \& Sons, 1958.

[2] P.R. Bevington, "Data Reduction and Error Analysis for the Physical Sciences”, McGraw-Hill Book Company, 1969.

[3] C.Y. Tan, J.M. Steimel,"The Effect of Noise on Transverse Emittance Growth in the Tevatron", Fermilab Technical Note, TM-2121, for more details. 